\title{
Abstracts of Papers Presented at the 117th Meeting of the American Astronomical Society, held 28-31 December 1964 at Montreal, Canada
}

The Polarization of the Thermal Radiation of the Moon at $14.5 \mathrm{GHz}$. J. W. M. BAars, P. G. Mezger, N. Savin, and H. WendKer, National Radio Astronomy Observatory.-In the spring of 1964, the thermal radiation of the moon was measured in two perpendicular polarization directions. As pointed out originally by Troitskii and Zeitlin (Radiofisica 3,1127, 1960), it is possible to derive the dielectric constant $\epsilon$ of the surface layer of the moon from these measurements.

From the measured drift and declination scans we derived the emissivity in both polarization angles (normalized to unity in the center of the moon) and the tangential polarization (i.e. the ratio of the emissivity of a point on the surface in the two polarization directions).

In order to find the dielectric constant we computed the emissivity of a smooth sphere for values of $\epsilon$ separated by steps of 0.1 in the range $1.1 \leqslant \epsilon \leqslant 2.5 \mathrm{We}$ convolved these curves with the gaussian beam pattern of our 85-ft antenna (3.35 arc beamwidth) and derived the tangential polarization.

As a next step we assumed a large-scale surface roughness for the moon with the normals to the surface having a Gaussian distribution around the line of sight with a "half-width" of $5,10,15,20,25$, and $30 \mathrm{deg}$. The theoretical curves computed with these models were also convolved with the antenna beam.

The comparison of the computed model curves with the average of all our measurements shows that the role of the roughness is not serious in the central part of the lunar disk up to about 0.6 of the radius from the center. Within this radius $\epsilon=1.8$ gives the best fit to the measurements. In the region 0.6 to 0.9 of the radius, the roughness is important and a model with roughness of $15 \mathrm{deg}$ half-width and dielectric constant $\epsilon=1.5$ yields the best fit. Close to the edge of the moon at $>0.9$ of the radius from the center, the polarization decreases rapidly. This decrease may be explained by the more normal incidence of the radiation coming from the mountains near the lunar limb.

Our work, together with previously published values of the dielectric constant at different frequencies, suggests that the dielectric constant decreases with increasing frequency.

Search for Variable Stars in Galactic Clusters. Gustav A. Bakos, Dearborn Observatory.-The variable star in NGC 2682 (Fagerholm No. 65), discovered earlier, has now been tentatively classified as a flare star. Apparently, it is a nonmember of the cluster. A new variable has been found in NGC 6871. It is an RR Lyrae type star with a period of 0.275 days. Since the apparent magnitude is 13 the distance of the variable is about twice the distance of the cluster itself.

Geomagnetic Effects of Plage Areas on the Sun. R. Glenn Bateman, Yale College, and Ludwig Oster, Yale University Observatory.-A study was made of the relation between the central meridian passage of activity centers on the sun and the $K_{p}$ geomagnetic index in the years 1960-1963. We have confirmed Becker's (Z. Astrophys. 32, 195, 1953 ) earlier results that the CMP of two sunspot groups arranged symmetrically (same longitude, corresponding northern and southern latitude) is followed by a marked low in geomagnetic activity. The study has been extended to plage areas with and without sunspots which were neither followed nor preceded within three days by another activity center. The significance of this criterion is discussed. We found a pronounced minimum in $K_{p}$ occurring slightly over two days after the CMP of plage areas with latitudes up to $\pm 30^{\circ}$, with the minimum's half-width apparently being independent of the area's latitude. These results seem to exclude the possibility that the large-scale structure of the magnetic fields due to the mirror symmetry (Oster, Boeing Sci. Res. Labs. Rept. D1-82-0216, 1963) plays a significant role in the guiding of the agent responsible for the geomagnetic event. In fact, any causal relationship would involve the magnetic field of isolated centers of activity. This work was supported by the National Aeronautics and Space Administration.

The Brightness Distribution of Jupiter's 10 and $21 \mathrm{~cm}$ Radio Emission. G. L. Berge, Owens Valley Radio Observatory.-A study of the brightness distribution (including the polarization distribution) of Jupiter has recently been carried out at the Owens Valley Radio Observatory. Using the twin 90-ft paraboloids as an interferometer, observations have been made with various east-west baselines ranging from 300 to $4800 \lambda$ at $10.4 \mathrm{~cm}$ and 150 to $2400 \lambda$ at $21.2 \mathrm{~cm}$ and also with some crucial north-south baselines at $10.4 \mathrm{~cm}$. The results are in agreement with earlier size measurements which gave the polar and equatorial dimensions as one and three planetary diameters, respectively (Morris, D., and Berge, G. L., Astrophys. J. 136, 276, 1962). However, the new measurements are more complete and show much more fine structure.

The detailed results lead to the following conclusions: (1) The disk emission at $10.4 \mathrm{~cm}$ is about 